\title{
Artículos
}


$\frac{\overline{0}}{0}$

ह

응

$\xi$

8 


\section{Políticas Públicas y formación sibuada. Desafíos del distanciamiento}

C. Copyright 2021. Universidad Nacional Autónoma de Nicaragua, Managua (UNAN-Managua) Todos los derechos reservados

\section{Public Policies and situated training. Challenges of distancing}

Mariela Edelstein

Universidad Provincial de Córdoba

https://orcid.org/0000-0001-7583-8877

marielae@upc.edu.ar
Daniela Blasco

Universidad Provincial de Córdoba

https://orcid.org/0000-0003-1174-2221

danielablasco@upc.edu.ar
Fecha de recibido: $23 / 04 / 2021$

\section{Resumen}

Este artículo está pensado como un encuentro, una conversación, entre quienes están escribiendo y un convite a quienes lo lean para formar parte del diálogo, que puedan interpelar las palabras, acompañando estas ideas con experiencias propias. Se intenta generar una conversación desde la trama que habilita a las dimensiones educación, prácticas pre profesionales y extensionistas, contextos y políticas públicas.

Se hace referencia a las prácticas pre profesionales, aquellas que estudiantes de todos los campos desarrollan previo a su graduación, aunque enfocado principalmente en el área social y en el escenario educativo de la crisis sanitaria por pandemia. Con base a la experiencia que se desarrolló en el año 2020, se reflexionan las vivencias en el vínculo específico de las instituciones académicas - con sus docentes, investigadores, extensionistas y estudiantes - en relación a efectores específicos de políticas públicas. Esta experiencia parte de una articulación con un área puntual del Estado Provincial de Córdoba, en Argentina, la Secretaría de Niñez, Adolescencia y Familia.
Fecha de dictaminado: 22/05/2021

A partir de este análisis de caso, se establecen algunas vinculaciones entre el aspecto político de la educación y los necesarios diálogos de saberes entre universidades y territorios, en una trama que se pretende reflexiva.

\section{Palabras claves}

Educación, experiencias pre profesionales, políticas públicas, contexto.

\section{Summary}

This article is intended as a meeting, a conversation, between those of us who are writing and an invitation to those who read it to be part of the dialogue, who can question the words, accompanying these ideas with their own experiences. We try to generate a conversation from the plot that enables the dimensions of education, pre-professional and extension practices, contexts and public policies.

We refer to pre-professional practices, those that students from all fields develop prior to graduation, although mainly focused on the social field and on 
the educational scenario of the health crisis due to pandemic. We use the experience that the year 2020 allowed to develop and from those experiences we reflect on the specific link of academic institutions with their teachers, researchers, extension workers and students - in relation to specific effectors of public policies. This experience is based on an articulation with a specific area of the Provincial State of Córdoba, in Argentina, the Secretariat for Children, Adolescents and the Family.

From this case analysis, of which we are part, we establish some links between the political aspect of education and the necessary dialogues of knowledge between universities and territories, in a plot that is intended to be reflective.

\section{Keywords}

Education, pre-professional experiences, public policies, context.

\section{Introducción}

\section{El contexto del poder decir en un espacio de encuentros}

La Secretaría de Niñez, Adolescencia y Familia (SENAF) de la Provincia de Córdoba, nace en el año 2007 a partir de una decisión del Poder Ejecutivo Provincial, con el fin de generar las condiciones que posibiliten la efectiva implementación de Políticas Públicas que garanticen el acceso y la protección de derechos de niños, niñas y adolescentes de acuerdo con la Ley Nacional 26.061 a la cual la Provincia ya había adherido. A partir de entonces, se estructuran áreas administrativas en SENAF y se resuelve una organización territorial para dar cobertura a las distintas regiones de la provincia. Es en el año 2011 que se logra, luego de consensos y acuerdos con diferentes sectores y otros poderes del Estado legislar en Córdoba en esta línea y aprobar la Ley 9944.

Durante ese período transicional nos incorporamos en la escena sociopolítica con el desafío de gestionar los alcances de la Dirección de Capacitación y Coordinación Operativa, desde la que se asume como una de las tareas prioritarias realizar una propuesta de articulación con las universidades, a fin de poder avanzar en un diálogo necesario entre el Estado y la Academia. Se constituye una mesa de trabajo con representantes de las distintas disciplinas que en su formación profesional pueden realizar prácticas pre profesionales en la SENAF y la Secretaría establece, a su vez, espacios y referentes para el acompañamiento a estudiantes.

La iniciativa crece durante estos años de experiencias de trabajo colaborativo e interinstitucional sostenido y en el cierre de las prácticas del año académico 2020 se organizó, como cada año, el Encuentro Interdisciplinario de Practicantes Universitarios en SENAF, cuya apertura se desarrolló mediante una conferencia. Este trabajo surge de ese encuentro con una significación especial en la trama de la experiencia compartida, ya que fue el primero desarrollado en la virtualidad, dado el marco del particular contexto de pandemia mundial.

La experiencia del espacio de acompañamiento a las prácticas pre profesionales desde SENAF, hoy nos interroga en diferentes roles profesionales a ambas. En tanto docentes universitarias, como parte del equipo de SENAF y de la Secretaría de Extensión y Relaciones Institucionales de la Universidad Provincial de Córdoba. De esta manera, también se piensa este texto/trama como una experiencia dialógica.

Teniendo en cuenta que los textos y relatos producidos, se generan en contextos de situación y relaciones, este artículo se desarrolla en ese diálogo de temporalidades y actividades, en una reflexión que interpela los campos de las políticas públicas y la formación académica, con sus intersecciones. Este recorrido emprendido con otras personas en contextos institucionales, de alguna manera estructurado por esa institución, deja huellas que pueden recorrerse, nos marcan como profesionales, marcando una ruta y son parte de nuestro ser y estar en el mundo, como individuos y actores sociales.

Pensamos este artículo también como un encuentro, una conversación. La intención, al igual que tuvo la conferencia, es que los lectores, puedan reflexionar las palabras, acompañando las ideas con experiencias propias. Todos tenemos una conversación privada mientras acontece otra que se puede denominar pública, o mientras se produce el acto activo de una lectura, porque todo texto es una trama e implica a otras personas. De alguna manera, al leer acerca de las experiencias de otros se genera un diálogo con nosotras y nosotros mismos, que ocurre en simultáneo, y esto es una riqueza del lenguaje. 
Pueden ustedes, lectores y lectoras, tomar notas, también de esa conversación privada y de lo que esta genere, estaremos atentas al email para poder compartir con ustedes sus reflexiones.

\section{Contexto de situación}

El año 2020 no será uno más en el calendario de la humanidad, nos tocó vivir a todas las personas y en todas partes del mundo un quiebre, incluso, un cambio de época. La pandemia por COVID-19 llegó de un día para otro, con todo su alcance invisible y a todas partes casi en simultáneo. Como si no solo viajara con las personas sino también por internet. Este tiempo de híper comunicación, también impulsó de alguna manera la velocidad del virus. Incluso desde la información (o sobreinformación) de las corporaciones de medios de comunicación que nos llevaron a un sistema de contabilidad de vidas, camas críticas y personas "infectadas". Nos enfrentó con una gran cantidad de desafíos, instalándose en tanto crisis epidemiológica, pero también de sentido. Este artículo no pretende desarrollar el tema, porque es de lo que se habla todos los días en el mundo entero, pero no se puede evitar pensar en contexto. Este es nuestro tiempo histórico, por lo que se vuelve fundamental situarnos para la reflexión.

Las universidades, al igual que los demás espacios y niveles del sistema educativo, debieron virtualizar sus vínculos pedagógicos. Así sucedieron clases, conferencias y debates mediados por pantallas, se tuvo que dejar de circular por aulas y pasillos, los edificios escolares quedaron sin personas, aunque las asignaturas, de todas maneras, ocurrieron; cada una desde su casa y a la vez cada clase en un cotidiano de encuentros tecnológicos. No circularon las personas, pero sí las palabras.

Otra cuestión que es importante traer a la reflexión, es el hecho que, nos hemos apropiado de una tecnología que no nos pertenecía, que la usábamos para leer o escuchar la palabra de otras personas, de algunos catedráticos, de "yutubers", pero no era nuestra palabra la que circulaba por internet, ahora sí. Este contexto nos invitó a formar parte de un medio, a tomar la palabra nuevamente, de cierto modo, se democratizó el poder de hablar. Entonces, otra situación que caracterizó al 2020, fue la apropiación que hicimos de distintos canales de comunicación, de modo que, el aislamiento fue realmente físico y no social para una cantidad de personas que tuvimos posibilidades de acceso a la tecnología. Pero, justamente, ese mismo derecho (que en la realidad se materializó en tanto privilegio) puso en evidencia distanciamientos (abismales) entre quienes no acceden a los recursos y quienes sí. Se potenciaron inequidades, desigualdades, injusticias, problemas estructurales que suelen estar ocultos, se naturalizan, pero quedaron a mayor descubierto a raíz de la pandemia.

También, este desorden que se produjo en las estructuras de las instituciones que se conocen, por las que se circulan, de las que se forma parte, nos cuestionó como individuos, pero, fundamentalmente, en tanto profesionales, ciudadanos y actores sociales, desde esta misma crisis de sentido, por lo que nos encontramos ante el desafío de volver a construir categorías, re definir y pensar (nos) de nuevo.

Durante el 2020 (y se continúa este año), las y los estudiantes, docentes, trabajadores y trabajadoras, pero también los niños, las niñas y los adolescentes que tenían bien aprendido el "oficio" de habitar las escuelas, tuvieron una experiencia mediatizada de educación, radicalmente diferente a la que se tiene registrada, la que formaba parte del habitus. En un corto período de tiempo todos y todas, sin importar edades, atravesaron una transformación de esquemas sin referencias previas, desde la simple convicción de que la circulación de los bienes simbólicos de la cultura, divulgada por los formatos escolarizados -los nuevos ahora- resultaba una cuestión impostergable.

\section{Algunas consideraciones sobre lo político y las políticas públicas}

En este apartado no se pretende enunciar definiciones sino compartir una parte de nuestra experiencia, algunas de las reflexiones que formaron y forman parte de esta historia, que nos es común. Se hace referencia a las experiencias en el sentido que le otorga Larrosa (2018), quien las plantea como "eso que nos pasa, y no simplemente eso que pasa". Si bien son acontecimientos exteriores a las personas, es por medio de las vivencias que cobran sentido.

La experiencia supone, en primer lugar, un acontecimiento o, dicho de otro modo, el pasar de algo que no soy yo. $Y$ "algo que no soy yo" significa también algo que no depende de mí, que no es una proyección de mí mismo, que no es el resultado de mis palabras, ni de mis ideas, ni de mis representaciones, ni de mis sentimientos, ni de mis proyectos, ni de mis 
intenciones, que no depende ni de mi saber, ni de mi poder, ni de mi voluntad. "Que no soy yo" significa que es "otra cosa que yo", otra cosa que lo que yo digo, lo que yo sé, lo que yo siento, lo que yo pienso, lo que yo anticipo, lo que yo puedo, lo que yo quiero. (Larrosa, 2018, p. 2)

En este sentido se vincula la idea de lo político a lo experiencial, a la vivencia de las personas en el marco de recorridos institucionales, que en este caso se establecen entre un espacio del gobierno de la Provincia de Córdoba y los de formación académica, entre trabajadores y trabajadoras del Estado, docentes, y estudiantes de carreras universitarias. Desde esta participación, asumimos un rol político como profesionales y a su vez las instituciones que constituye también lo representan. También se pretende hacerse cargo de ello y trabajar colectivamente para que este rol se desempeñe en colaboración y responsablemente, con un objetivo de futuro y en miras a construir buenas y mejores prácticas.

Muchas veces se escucha hablar de políticas públicas como si fuera alguna cosa con entidad propia, algo que ocurre más allá de las personas, y las instituciones. O bien, se las suele pensar como un plan establecido por quienes están en determinadas posiciones de poder, que ocupan ciertos espacios en unas estructuras inamovibles. Ellos, que siguen determinados intereses, pero no siempre coinciden con los de la gente, los del "pueblo" al que se considera en tanto "destinatario", "beneficiario" u objeto de esas políticas.

Entendemos que desde dicho imaginario colectivo a lo político y las políticas públicas se les asignan una gran variedad de connotaciones negativas, generadoras de disputas. En el contexto de esta reflexión, como para poner en la ronda la forma en que apelamos a ciertos términos, sin ánimo de entrar en definiciones sino más bien como para no caer en trampas semánticas, podemos decir que asumimos lo político como atributo de la humanidad. Hay quienes relacionan lo político con la posibilidad de lo instituyente, y en ese sentido nos gusta devolver a la palabra la fuerza de los procesos necesarios para la toma de decisiones (máxime si hablamos de derechos de NNyA). Aristóteles, ya consideraba que lo político es lo que diferencia al ser humano del animal, entonces, desde ese lugar general, consiste en la capacidad de dar forma a la vida social, se fundamenta en el carácter relacional de las personas y se vincula con los proyectos colectivos. Por otro lado, Hannah Arendt nos acerca esta afirmación a la que siempre apelamos cuando intentamos dar cuerpo a las ideas en torno a este tema, ella plantea que el mundo de lo político es, un estar juntos siendo distintos:

La política se basa en el hecho de la pluralidad de los hombres (...) se trata del estar juntos y los unos con los otros de los diversos (...) la participación activa, en la pluralidad. (Arendt, 1997, p.45-46)

De esta manera, pensamos a lo político como el ámbito de la palabra, el consenso, el acuerdo, las relaciones cooperativas y las disputas de poder hasta lograrlo. Así, la actividad política consiste en conciliar, por medio de la palabra, las diferencias y que resultan de la diversidad, con el propósito de vivir en armonía. (Arendt; 1997). En el entorno de esta conversación, que pretende tejer reflexiones en torno a los procesos de formación situados, acercamos también al diálogo a Paulo Freire, quien nos recuerda la esencia política de las prácticas educativas:

No hay práctica educativa que no sea política; no hay práctica educativa que no esté envuelta en sueños y no hay práctica educativa que no involucre valores, proyectos, utopías. No hay entonces práctica educativa sin ética. (Freire, 2014, p. 50-51)

Desde nuestra formación académica, práctica profesional y filiaciones institucionales compartidas siempre nos ocupó el intento de vincular la práctica en el Estado, el ejercicio del diseño de acciones, proyectos, programas y políticas públicas con reflexiones académicas. Por lo general, suele habitarse un marcado distanciamiento, a veces abismal, entre los profesionales que forman parte del Estado y quienes se desempeñan en el mundo académico. Pero también es cierto que los y las profesionales que trabajan en el Estado, estudiaron en universidades, se formaron como profesionales y luego empezaron a trabajar o incluso completaron sus estudios mientras trabajaban, entonces a causa de ese punto de origen es que la distancia es ficticia, aunque en el territorio educativo, se suele transitar uno y otro escenario en momentos diferenciados. Ocurre con demasiada frecuencia que el momento del aprendizaje se relaciona con el aula y el momento del ejercicio profesional con las instituciones y las comunidades. Se disocia fácilmente el saber, del ser, el estar y el hacer que requiere de manera integral el proceso de formación (permanente). 
Se debe asumir la tarea de una revinculación y las responsabilidades que para ello son compartidas. La Academia debe permitirse una permeabilidad con las políticas públicas y el Estado, nutrir sus propuestas y políticas de análisis compartidos, reflexiones interdisciplinarias y en ese sentido favorecer el ingreso de estudiantes, de personas en formación que aporten esa mirada de recién llegados que tanto bien les hace a las instituciones.

En este punto, se considera necesario establecer algunos acuerdos sobre qué entendemos por políticas públicas ya que es uno de los conceptos más polisémicos del campo de las ciencias sociales, de acuerdo a la CEPAL (Comisión Económica para América Latina y el Caribe).

Las políticas públicas son soluciones específicas de cómo manejar los asuntos internos que, se desprenden de la agenda estatal. De alguna manera, son las que permiten orientar la gestión de un gobierno para alcanzar sus objetivos (y en los sistemas democráticos esos objetivos serían los motivos por los que la gente les dio el voto). Es por ello que el diseño, gestión y evaluación de las políticas públicas son una parte fundamental del quehacer de los gobiernos (Lahera, 2004, p. 7-10).

El Banco Mundial define a una política específica como un plan para alcanzar un objetivo de interés público, y esta conceptualización desde el análisis propuesto por la CEPAL se amplía haciendo referencia a un conjunto de actividades (programas, estrategias, procedimientos, leyes, reglamentos) dirigido hacia un objetivo general. Estas actividades frecuentemente se acumulan durante años. La política pública se diferencia de un programa en cuanto a que es una intervención directa sobre una realidad social, usada, en conjunto con otros programas (y otros instrumentos de política), para ser implementada.

También forman parte de las políticas públicas, las instituciones del estado que se establecen como ejecutoras. Podemos pensar en ejemplos, seguramente, ustedes lo están haciendo. Para hacer referencia al ámbito de las infancias, la Convención Internacional de Derechos de los niños y las normativas nacionales que cada país estableció para dar cumplimiento al compromiso mundial, las Leyes 26061 de Argentina y la 9944 de Córdoba a las que hicimos referencia, forman parte de una política pública de Argentina en relación al necesario establecimiento de un enfoque de derechos para la atención de diferentes problemáticas que atraviesan las infancias, en ese contexto también se determina que el órgano de aplicación para estas leyes será la SENAF. Es decir, el espacio del Estado que tendrá a cargo el diseño, ejecución y evaluación de acciones, proyectos y programas específicos, responsable de establecer una política pública, al mismo tiempo que se establece la corresponsabilidad como principio determinante en su construcción y consolidación. Es interesante, no desconocer esta idea-fuerza que nos mantiene en alerta de la necesaria integralidad en la atención de las problemáticas sociales, y por lo tanto nos incluye en tanto universidad.

\section{Prácticas situadas}

Ahora bien, ¿por qué plantearse el vínculo entre las políticas públicas y la formación situada?

Las y los estudiantes de carreras universitarias, mientras transcurren su experiencia educativa, con mayor o menor nivel de formalidad realizan prácticas pre profesionales. Estas pueden adoptar la forma de un trabajo final para graduarse, una materia en la carrera, como parte de un proyecto específico de investigación o de extensión. Se considera a la práctica como un espacio fundamental de formación, en el cual desarrollamos el impostergable diálogo entre los saberes académicos y los saberes populares, de las y los vecinos de la barriada, de los niños y las niñas, reales, locales, con familias y comunidades, en territorios concretos.

Desde nuestro punto de vista, es imposible pensar en una práctica no situada, no obstante, en muchas carreras universitarias se realizan simulaciones de espacios y problemáticas sociales - trabajo sobre situaciones hipotéticas - y nunca, hasta el momento de graduarse, se proponen vínculos con esa realidad concreta a la cual se pretende conocer. Por lo tanto, sí encontramos en la formación de grado numerosos ejemplos.

Partimos aquí de unacuerdo de base, estas prácticas que se desarrollan en situaciones reales, en territorios (no universitarios) son también contextos de formación, con la particularidad que implican un espacio y otro tiempo. Distinto al del aula y el calendario académico, pues se sale de las horas cátedra y del turno que se cursa. En este momento educativo de pandemia, también se distanciaron de todos los otros espacios 
territoriales y los habitaron desde una mediatización tecnológica que todavía no se logra procesar, aunque sí se puede afirmar, que como parte de estos nuevos procesos se debió colaborar comprometidamente la creación de nuevas estrategias y el acompañamiento de estudiantes que reacomodaron sus pre conceptos a estas nuevas formas. No fue sencillo, aunque sí, un proceso que devino en una experiencia fructífera.

Diferentes expresiones remiten a los procesos de prácticas que se denominan como profesionales y pre profesionales, formación situada, trabajo en territorio, salida al campo, entre otros modos de nombrarlas. Nosotras, siempre vamos a adherir a un acuerdo, más allá de su conceptualización, entendemos a la práctica como un intercambio, un diálogo. Nuestro compañero Ezequiel Kowalsky - educador comunitario - en el seminario de Extensión Universitaria de la UPC introdujo un concepto interesante para referir a los intercambios de saberes, nos habló del convite noble y aquí es valioso reflexionar que este convite se desarrolla de manera dinámica y en retroalimentación permanente ¿quién convida a quién?

En un proceso formativo, entonces, se involucran actores diversos. Se implican las instituciones que incluso se denominan, "co formadoras". Ponemos aquí en tensión este nombre, ya que desde nuestro enfoque seríamos un colectivo dinámico de co formadores. Incluso desde los espacios del Estado que reciben a estudiantes, en este caso la SENAF, la presencia de practicantes en las instituciones o programas también tiene una intencionalidad formativa hacia adentro del mismo. Es decir, las instancias académicas se renuevan con la mirada de los "recién llegados" o "extranjeros".

Dicen Graciela Frigerio y Gabriela Diker (2003) acerca de la figura del extranjero en educación que:

Lo extranjero provoca miedo, pero al mismo tiempo atracción. En lo pedagógico se habla del profesor como extranjero para el alumno; cada alumno, cada grupo, también son extranjeros al profesor. La noción de extranjero irrumpe en la historia, en la literatura, en las ciencias, en la educación. Lo extranjero es uno de los nombres de la alteridad; es un argumento, un punto de partida, una alegoría, un goce, una mirada.

Nos parece una metáfora bien interesante pensar en quien está realizando una práctica como extranjero en este sentido de alteridad y es una invitación que les hacemos a estudiantes y docentes que transitan el espacio de la práctica.
Por otro lado, compartiendo el análisis que realiza Chapato María Elsa (UNICEN 2016, p. 2-9), en estos escenarios de prácticas, circulan deliberadamente y como condición implícita de su propia razón de ser, reflexiones y conocimientos. Los períodos de prácticas en tanto experiencias de formación situada, se constituyen en oportunidades para hacer posible la circulación de ideas y pensamientos sobre los problemas que detectamos, donde se produzcan confrontaciones teóricas entre lo que sabíamos o creíamos saber y nuevas posturas que van perfilándose en el horizonte del trabajo académico, que nos interpelan en nuestros viejos saberes y reclaman nuevas elaboraciones, nuevos posicionamientos, nuevas respuestas.

Las prácticas de formación situadas en contextos cambiantes, multidimensionales y cada vez más complejos, son desafíos intensos y extensos. Y la propuesta que se realiza desde la SENAF, en tanto escenario y escena compleja que abre puertas de instituciones y programas para dar lugar y dejarse interpelar e interpretar, por esta suerte de extranjeros, es una experiencia que no se puede vivenciar en muchos espacios del estado o en otras políticas públicas, porque es un propósito de la SENAF, y desde ese lugar de la intencionalidad se construye y se crece en conjunto.

Estas prácticas se constituyen en modos específicos de aprendizaje, ya que se realizan en tanto un proceso individual como todo aprendizaje, pero al tener lugar en interacciones entre personas que se vinculan desde diferentes posiciones, se vuelve social, societal podríamos también decir, porque ocurre en espacios que son formados o "con formados" por diferentes actores de las instituciones. En estas condiciones podemos aprender acerca de las prácticas humanas que a su vez remiten a acciones concretas de sujetos que actúan en contextos sociales significativos, relacionales.

Así, siguiendo el análisis de Chapato (op. Cit), cuando se pretende comprender las prácticas de sujetos particulares debemos atender a que los contextos no son ámbitos físicos o solo espacios institucionales que ponen en evidencia un devenir histórico, sino que los entendemos como sistemas de actividades en los que se inserta la acción particular de un sujeto (una persona) que realiza operaciones cuyos sentidos sólo pueden comprenderse en el marco del sistema general de acción. Las acciones individuales, deben 
ser interpretadas teniendo en cuenta vicisitudes y factores que no están inmediatamente presentes en la situación, ni contenidos exclusivamente en las personas que actúan en esas situaciones.

Son esos contextos y las relaciones sociales que ahí se despliegan las que permiten alcanzar una mayor y más compleja interpretación de las situaciones y las decisiones $\mathrm{u}$ operaciones que las personas realizan en este tiempo y espacio específicos (Jara, 2018), nos habla de estos intercambios en términos de experiencias vivenciales:

La experiencia es siempre vivencial: implica una vinculación física, emocional y también intelectual con el conjunto de elementos del entramado inmediato con el que me relaciono. Las experiencias son, por tanto, lugares vivos de creación y producción de saberes. (p.54).

Este modo de concebir el aprendizaje y su relación contextual, el hecho de que las prácticas sean situadas, nos permite enriquecer nuestras comprensiones y realizar inferencias sobre los guiones sociales en que se incluyen las actividades, dado que ya todos habíamos percibido que las acciones de los sujetos tienen sentido en los contextos de acción en que se realizan, devienen del desarrollo histórico de esos contextos, de la propia historia de los sujetos y del sentido social atribuido a las acciones, a los propios sujetos practicantes e instituciones o tradiciones que los contienen.

Podemos pensar también, que las instituciones contienen en su seno procesos instituidos, mandatos, lógicas de trabajo, una cultura que le son propias, y brindan su identitario. Incluirse en estos procesos y hacerlo con el invite, mediante el acompañamiento de quienes forman parte, es un proceso bien interesante para quienes están formándose como profesionales de alguno de los campos que están en juego. Creemos que es esto, un juego, un diálogo, una conversación.

\section{Conclusiones}

Hay instituciones que de tanto repetirse a sí mismas se pueden ver como cerradas, difíciles de adaptar, las que forman parte de SENAF vienen de unos mandatos y funciones instituidas muy fuertes y por eso se presentan de ese modo. Sin embargo, al abrir las puertas a los espacios de práctica demuestran un valor o deseo de cambio. No es fácil, hay mucha historia, un fuerte imaginario social que deconstruir. Son, en muchos casos, lógicas organizativas y de cierta preeminencia de técnicas y recursos, convertidos en convencionales y legitimados por la fuerza de las tradiciones, generalmente no interrogadas.

Se ponen en juego una multiplicidad de dimensiones y elementos al pensar en estas prácticas, algunas se relacionan con la temporalidad, con ciertos elementos materiales (presentes o ausentes), con el entorno natural, la historia de las personas que habitan cada espacio y las historias mismas de esos espacios. También tenemos que considerar los aspectos discursivos y narrativas, entre otras tantas cuestiones estructurales y simbólicas que no solo están relacionadas con las prácticas específicas, relaciones entre sujetos situados, sino también que impactan en las políticas públicas de las que son invitadas a formar parte.

El punto de vínculo aquí se nos presenta dinámico. $Y$ esta pandemia, que nos puso a reflexionar sobre muchos de los sentidos establecidos, los cómodos, los incómodos, los seguros y los inciertos. Este es un excelente momento para plantearnos otras prácticas posibles, que no serán individuales, que no serán unidireccionales, que serán seguramente colectivas e interdisciplinares. Se asumirán formas y estarán mediatizadas por diferentes estructuras ya que no sólo las pantallas nos informan.

Es un tiempo para pensarnos como parte de las políticas públicas que estamos conociendo, que por el mismo hecho de hacerles la visita desde la práctica ya podemos observar y desde ese lugar observarnos. También la invitación será a volver a visitar los conceptos y teorías dominantes en las carreras que hemos elegido para desarrollarnos individual y colectivamente, releyéndolas nuevamente.

En síntesis, podemos decir que una práctica situada es hacerse preguntas distintas, no las mismas que surgen en las aulas, porque seguramente, preguntas diferentes nos podrán llevar a caminos distintos de búsqueda. 


\section{Referencias bibliográficas}

- Arendt, H. (1997). ¿Qué es política? Barcelona: Paidós.

- Arendt, H. (2009). La condición humana. Buenos Aires: Paidos.

- Chapato, M. E. (2016). El abordaje de la práctica de enseñanza situada: la captación de la complejidad. En R. UNICEN (Ed.), El abordaje de la práctica de enseñanza situada: la captación de la complejidad. (págs. 1-11). Tandil: UNICEN (Universidad Nacional del Centro de la provincia de Buenos Aires). Obtenido de http://www.ridaa.unicen.edu.ar/ xmlui/handle/123456789/468

- Freire, P. (2014). El grito manso. Buenos Aires: Siglo Veintiuno.

- Frigerio, G., \& Diker, G. (2003). Educación y alteridad. Las figuras del extranjero. Psicología y Educación, ensayos y experiencias, 48(48), 160166.

- Jara, O. (2018). La sistematización de experiencias: práctica y teoría para otros mundos posibles (1 ed.). Bogotá: CINDE.

- Lahera, E. (10 de Agosto de 2004). Política y políticas públicas en los procesos de reforma en América Latina. "Similitudes y diversidades" (Proyecto FRA/02/073). (N. Unicas, Ed.CEPAL. Serie Políticas Sociales, 1-32. Obtenido de https://www.fundacionhenrydunant. org/images/stories/biblioteca/PoliticasPublicas/Pol\%E2\%94\% 9 C\%C2\%A1tica\%20 y \% 2 o p ol \% E $2 \% 94 \%$ g C \% C $2 \%$ A 1 t i c a s \% 20 p\%E2\%94\%9C\%E2\%95\%91blicas.pdf

- Larrosa, J. (2018). Sobre la experiencia. (D. d. Primaria, Editor) Obtenido el 2021, de CEIP: http://www.ceip.edu.uy/documentos/2018/ifs/ dapg/materiales/Jorge_Larrosa_Experiencia_y_ alteridad.pdf 